\title{
Sarcopenia y fragilidad en sujetos sometidos a hemodiálisis en un centro de diálisis en el Perú
}

\author{
Sarcopenia and frailty in subjects undergoing \\ hemodialysis in a dialysis center in Perú
}

\author{
Brian Wally Mariños Cotrina ${ }^{*}$, Franco Salvador Rodríguez Brown Agurto², \\ Daniel Fernando Mendez Carbajal ${ }^{2}$
}

Recibido: 10 de noviembre de 2018. Aceptado para publicación: 15 de enero de 2019

Publicado en línea, enero 21 de 2019

https://doi.org/10.35454/rncm.v2n1.060

\section{Resumen}

Introducción: la toxemia urémica, la inflamación y las alteraciones metabólicas de sujetos con enfermedad renal crónica en hemodiálisis favorecen la sarcopenia y fragilidad. El objetivo de este estudio es determinar la prevalencia de sarcopenia y fragilidad en sujetos sometidos a hemodiálisis en Perú.

Métodos: estudio trasversal realizado en un centro de diálisis de Perú en noviembre de 2018. La muestra estuvo conformada por 111 pacientes con enfermedad renal crónica en terapia de hemodiálisis. Se empleó el cuestionario SARC-F para identificar el riesgo de sarcopenia, la escala clínica de fragilidad para detectar el riesgo de fragilidad y el fenotipo de Fried para el diagnóstico de fragilidad.

Resultados: se encontró riesgo de sarcopenia en $45,9 \%$ de los pacientes, riesgo de fragilidad en 19,8 \%, 46,8 \% de prefragilidad y $51,4 \%$ de fragilidad. Las mujeres presentaron mayor porcentaje de fragilidad mientras los hombres mayor porcentaje de riesgo de sarcopenia. El 17,1 \% de los pacientes estudiados presentó en forma concomitante sarcopenia y fragilidad.

Conclusiones: la prevalencia de sarcopenia y fragilidad en pacientes en hemodialisis es alta. En la población estudiada 1 de cada 2 sujetos está en riesgo de sarcopenia y tiene el diagnóstico de fragilidad. La presencia simultánea de ambas condiciones, sarcopenia y fragilidad, se observó en un porcentaje importante de pacientes.

Palabras clave: sarcopenia, fragilidad, SARC-F, escala clínica de fragilidad, diagnóstico de fragilidad.

\footnotetext{
1 Hospital Guillermo Kaelin de la Fuente, Lima, Perú.
}

\section{Summary}

Introduction: Uraemic toxemia, inflammation and metabolic alterations of subjects with chronic kidney disease in hemodialysis favors sarcopenia and / or frailty. The aim of this study was to determine the prevalence of sarcopenia and frailty in subjects undergoing hemodialysis in Peru.

Methods: The cross-sectional study was realized in a dialysis center of Peru in November of 2018. The sample consisted of 111 patients with chronic kidney disease on hemodialysis therapy. The SARC-F questionnaire was used to classify the risk of sarcopenia, the clinical frailty scale to detect the risk of frailty and the Fried's phenotype was used to diagnose of frailty.

Results: The risk of sarcopenia was found in $45.9 \%$ of patients; risk of frailty in $19.8 \%, 46.8 \%$ of prefrailty and $51.4 \%$ of frailty. Women showed more a higher percentage of frailty while men had higher risk of sarcopenia. $17.1 \%$ showed concomitantly sarcopenia and frailty.

Conclusions: The prevalence of sarcopenia and frailty in patients undergoing hemodialysis is higher. In the analyzed population, 1 of every 2 subjects is at risk of sarcopenia and has the diagnosis of frailty. Also, it is noteworthy that both conditions, sarcopenia and frailty, were present in a significant percentage of patients.

Key words: Sarcopenia; Frailty; SARC-F; Clinical frailty scale; Frailty diagnose.

\footnotetext{
2 Escuela de Nutrición de la Universidad Nacional Federico Villarreal Lima, Perú.
} 


\section{INTRODUCCIÓN}

La enfermedad renal crónica terminal se caracteriza por múltiples condiciones clínicas que empeoran el estado de salud como son la toxemia urémica, inflamación, alteraciones óseo minerales y del medio interno, entre otras ${ }^{(1)}$, aunado al catabolismo crónico producto de la terapia de reemplazo renal ${ }^{(2)}$. Esto altera el estado anatomo-fisiológico, que resulta en efectos deletéreos sobre la masa muscular, capacidad cognitiva y funcional. Es de notar que el estado inflamatorio/catabólico conlleva a la atrofia de la fibra muscular y disminución de la función mitocondrial a través de la activación de ciertos genes conocidos como atrogenes, que a su vez son el punto de partida para la síntesis de señales activadoras de autofagia y del sistema ubiquitina proteosoma $^{(3)}$. La autofagia, proceso fisiológico encargado del reciclado celular se ve incrementado por las citoquinas proinflamatorias lo que genera la destrucción mitocondrial y la consecuente muerte celular acelerada ${ }^{(4)}$. Al mismo tiempo, el sistema ubiquitina proteosoma degrada las miofibrillas ${ }^{(5)}$ produciendo atrofia muscular y disminución de la calidad del músculo ${ }^{(6)}$.

En consecuencia, estos sujetos son proclives a padecer ciertas alteraciones relacionadas con el sistema músculo esquelético y asociadas al estado nutricional. Dentro de estas se encuentran la sarcopenia ${ }^{(7)}$ y la fragilidad ${ }^{(8)}$.

Diferentes sociedades dedicadas al estudio de esta condición describen la sarcopenia como un síndrome caracterizado por la depleción de masa muscular, disminución de la fuerza y la función muscular ${ }^{(9,10)}$.

De acuerdo con su etiología, la sarcopenia se puede clasificar en dos categorías; primaria, que se encuentra ligada al avance de los años y es exclusiva del proceso de envejecimiento, mientras que la sarcopenia secundaria es el resultado de varios factores concomitantes incluidos la disminución de actividad física, patologías y nutrición, independiente de la edad del sujeto ${ }^{(11)}$.

En adición, la fragilidad es otra condición que refleja el deterioro de la calidad de vida del ser humano y se define como el estado de no resiliencia frente a los factores de estrés ${ }^{(12)}$, y al igual que la sarcopenia, se clasifica en primaria asociada al envejecimiento y secundaria, a la producida por enfermedades. El resultado de estas dos condiciones es la probabilidad de muerte ${ }^{(13,14)}$.

De lo mencionado anteriormente, se deprende la idea de que los sujetos con enfermedad renal crónica en hemodiálisis (ERCHD) pueden evidenciar sarcopenia y fragilidad según lo reportado en la literatura actual, evidenciándose la importancia de la prevención a través de su tamizaje y diagnóstico temprano. Además, en Perú no hay estudios que definan la frecuencia de sarcopenia ni fragilidad en esta población. Por esta razón, la presente investigación tiene el objetivo de determinar la prevalencia de sarcopenia y fragilidad en sujetos atendidos en un centro de diálisis en el Perú.

\section{MATERIALES Y MÉTODOS}

\section{Diseño del estudio}

Estudio de tipo descriptivo observacional de corte transversal el cual involucró a sujetos adultos jóvenes y adultos mayores sometidos a hemodiálisis. Se recolectaron los siguientes datos de la historia clínica: edad, tiempo de diálisis en meses, etiología de ERCHD. Los parámetros de riesgo de sarcopenia, riesgo de fragilidad y diagnóstico de fragilidad, fueron evaluados previo al tratamiento de diálisis.

\section{Participantes del estudio}

Este estudio involucró a todos los sujetos que recibieron terapia de hemodiálisis tres veces por semana distribuidos en ocho turnos diferentes en la clínica Hemobalance ubicada en la provincia constitucional del Callao, Perú.

\section{Riesgo de Sarcopenia}

El riesgo de sarcopenia fue valorado mediante la aplicación del cuestionario SARC-F ${ }^{(15)}$. Esta es una herramienta validada y sensible que analiza subjetivamente 5 componentes relacionados con la sarcopenia: fuerza, asistencia en la caminata, levantarse de una silla, subir escaleras y número de caídas en el último año. Cada componente presenta un puntaje máximo de 2 . Si la suma total del puntaje es igual o mayor a 4. Por defecto, si la suma es menor se concluye que la persona no presenta riesgo de sarcopenia.

\section{Riesgo de fragilidad}

El riesgo de fragilidad fue valorado a través de la escala clínica de fragilidad $^{(16)}$ (de los términos en inglés, clinical frailty scale o CFS) se utiliza el juicio subjetivo del evaluador, donde categoriza 9 estadios a través de una escala pictórica desde excelente condición física hasta enfermo en estado terminal. 


\section{Diagnóstico de fragilidad}

Para encontrar el diagnóstico de fragilidad se utilizó el formato del fenotipo de Fried et al. ${ }^{(17)}$ a través de 5 criterios: pérdida involuntaria de peso seco (mayor a $4,5 \mathrm{~kg}$ en el último año), presencia de fatiga mayor a 1 día en la semana, actividad física disminuida, debilidad de prensión manual y lentitud de la marcha. Este instrumento permite clasificar 3 estadios: si no cumple con ningún criterio, normal; si cumple con 1 o 2 criterios, prefrágil. Si cumple con 3 o más criterios, frágil. La debilidad de prensión manual se evaluó a través de dinamometría (dinamómetro manual digital marca CAMRY) por debajo del valor del P30 según Schlüssel et al. ${ }^{(18)} \mathrm{en} \mathrm{la}$ mano dominante o mano correspondiente al brazo sin presencia de fístula o catéter de alto flujo.

\section{Análisis estadístico}

Se utilizó el paquete estadístico SPSS versión 24 para el procesamiento y análisis de la información. Las características demográficas de los pacientes fueron descritas empleando distribuciones porcentuales para variables categóricas; mientras que las variables numéricas se utilizaron: medias, valores mínimos y máximos, desviación estándar e intervalos de confianza al $95 \%$.

\section{Consideraciones éticas}

El estudio se ajusta a la normativa ética nacional y sigue los principios éticos de la declaración de Helsinki. Además, este estudio se conceptualiza como una investigación sin riesgo debido a que solo se recolectan datos relacionados con cada uno de los sujetos sin ponerlos en situación de riesgo.

\section{RESULTADOS}

Se evaluaron 111 sujetos con tratamiento de hemodiálisis, donde $51,4 \%(\mathrm{n}=57)$ fueron mujeres y $48,6 \%(\mathrm{n}=54)$ hombres, siendo la mayoría adultos jóvenes $(66,7 \%)$. La media del tiempo de diálisis fue de 49,11 meses y las patologías más prevalentes que desencadenaron la enfermedad renal crónica fueron la Diabetes Mellitus $(35,14 \%)$ y la hipertensión arterial $(34,23 \%)$, como puede observarse en las tablas 1 y 2.

El riesgo de sarcopenia, según SARC-F, se encontró en $46,9 \%$ de los pacientes, donde más de la mitad fueron adultos mayores $78,4 \%(n=40)$. El riesgo de sarcopenia es más prevalente en adultos jóvenes y de sexo femenino (Figura 1).
Tabla 1. Características de la muestra

\begin{tabular}{|c|c|c|}
\hline Características & $\mathbf{n}$ & $\%$ \\
\hline \multicolumn{3}{|l|}{ Sexo } \\
\hline Masculino & 54 & 48,64 \\
\hline Femenino & 57 & 51,36 \\
\hline \multicolumn{3}{|l|}{ Edad } \\
\hline Adultos jóvenes & 75 & 67,57 \\
\hline Adultos mayores & 36 & 32,43 \\
\hline \multicolumn{3}{|l|}{ Etiología de la ERCHD } \\
\hline Diabetes Mellitus & 39 & 35,14 \\
\hline $\begin{array}{l}\text { Hipertensión arterial o Enf. de vasos } \\
\text { grandes }\end{array}$ & 38 & 34,23 \\
\hline Otras patologías & 14 & 12,61 \\
\hline Enf. Quística hereditaria congénita & 9 & 8,11 \\
\hline Nefritis Intersticial o Pielonefritis & 7 & 6,31 \\
\hline $\begin{array}{l}\text { Glomerulonefritis secundaria o } \\
\text { Vasculitis }\end{array}$ & 2 & 1,8 \\
\hline Neoplasias & 2 & 1,8 \\
\hline \multicolumn{3}{|l|}{ Riesgo de Sarcopenia } \\
\hline Con riesgo de sarcopenia & 52 & 46,85 \\
\hline Sin riesgo de sarcopenia & 59 & 53,15 \\
\hline \multicolumn{3}{|l|}{ Riesgo de fragilidad } \\
\hline Con riesgo de fragilidad & 22 & 20,72 \\
\hline Sin riesgo de fragilidad & 89 & 79,28 \\
\hline \multicolumn{3}{|l|}{ Diagnóstico de fragilidad } \\
\hline Normal & 2 & 1,80 \\
\hline Prefragilidad & 52 & 46,85 \\
\hline Fragilidad & 57 & 51,35 \\
\hline
\end{tabular}

Se encontró riesgo de fragilidad (según la CFS) en 20,7 \% de los sujetos analizados, siendo más prevalente en adultos mayores $86,4 \%(n=19)$ (Figura 2).

El porcentaje de sujetos evaluados que presen$\tan$ riesgo de sarcopenia y riesgo de fragilidad fue de $17,1 \%$ (Figura 3).

Fueron diagnosticados con fragilidad, según Fried, $54,1 \%$ de los pacientes; el $46,8 \%$, fueron diagnosticados prefrágiles, y 1,8 \% considerados normales. La fragilidad se presentó en mayor proporción en mujeres $56,1 \%(\mathrm{n}=32)$ y en adultos jóvenes $55,8 \%(\mathrm{n}=$ 29). Además, dentro del grupo de adultos mayores, las mujeres tuvieron mayor prevalencia de fragilidad $62,5 \%(n=25)$ con mayor porcentaje en mujeres (Figura 4). 
Tabla 2. Otros datos demográficos

\begin{tabular}{|l|c|c|c|c|c|c|c|}
\hline & $\mathbf{n}$ & Mínimo & Máximo & Media & Desviación & \multicolumn{2}{|c|}{$\mathbf{9 5 \% \text { IC }}$} \\
\cline { 4 - 8 } & & & & & & $\begin{array}{c}\text { Límite } \\
\text { inferión }\end{array}$ & $\begin{array}{c}\text { Límite } \\
\text { superior }\end{array}$ \\
\hline Edad & 111 & 24 & 87 & 61,95 & 12,811 & 59,55 & 64,36 \\
\hline Tiempo de diálisis (meses) & 111 & 0 & 219 & 49,11 & 39,631 & 41,65 & 56,56 \\
\hline
\end{tabular}

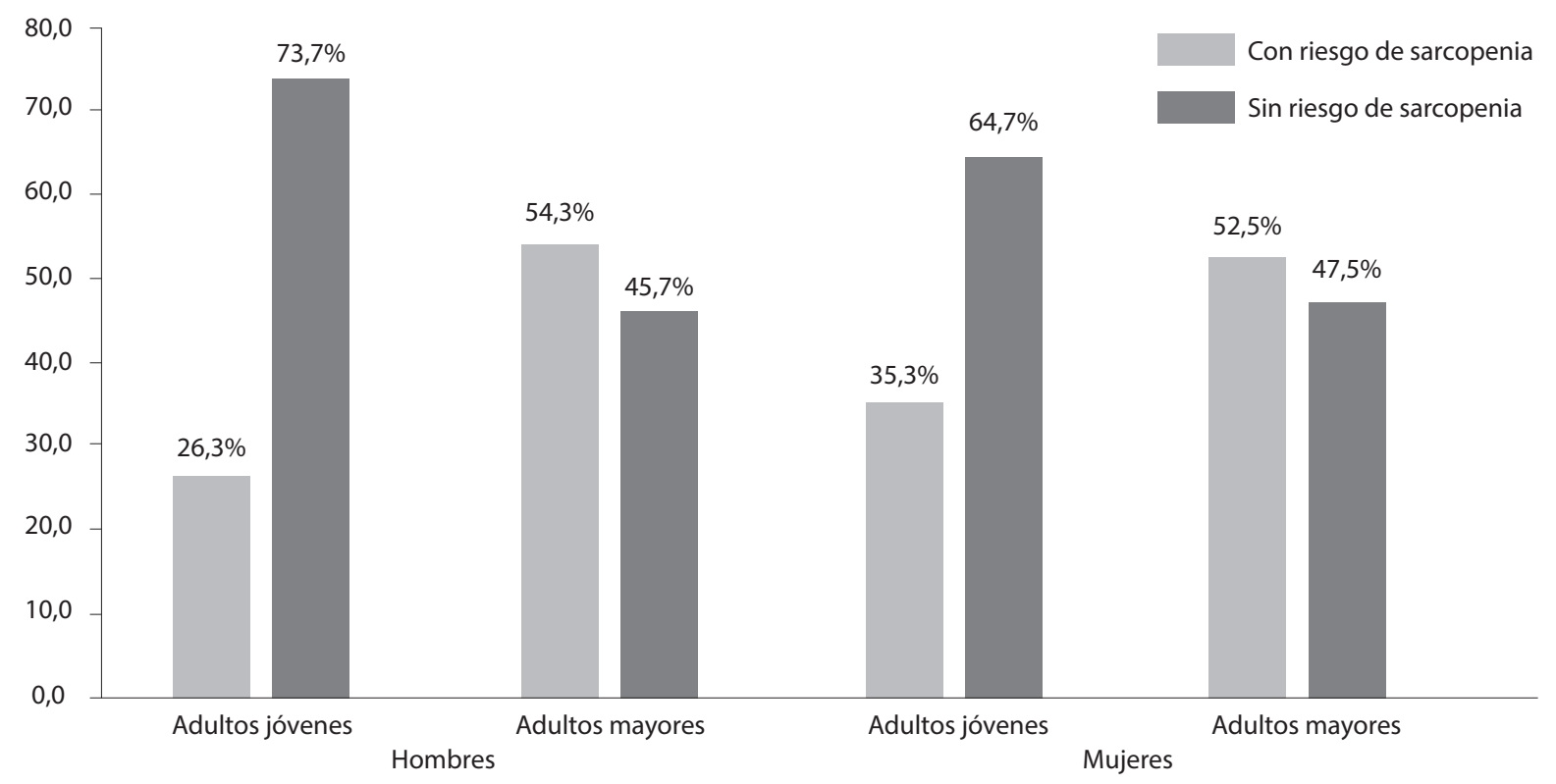

Figura 1. Riesgo de Sarcopenia en sujetos sometidos a hemodiálisis según edad y sexo.

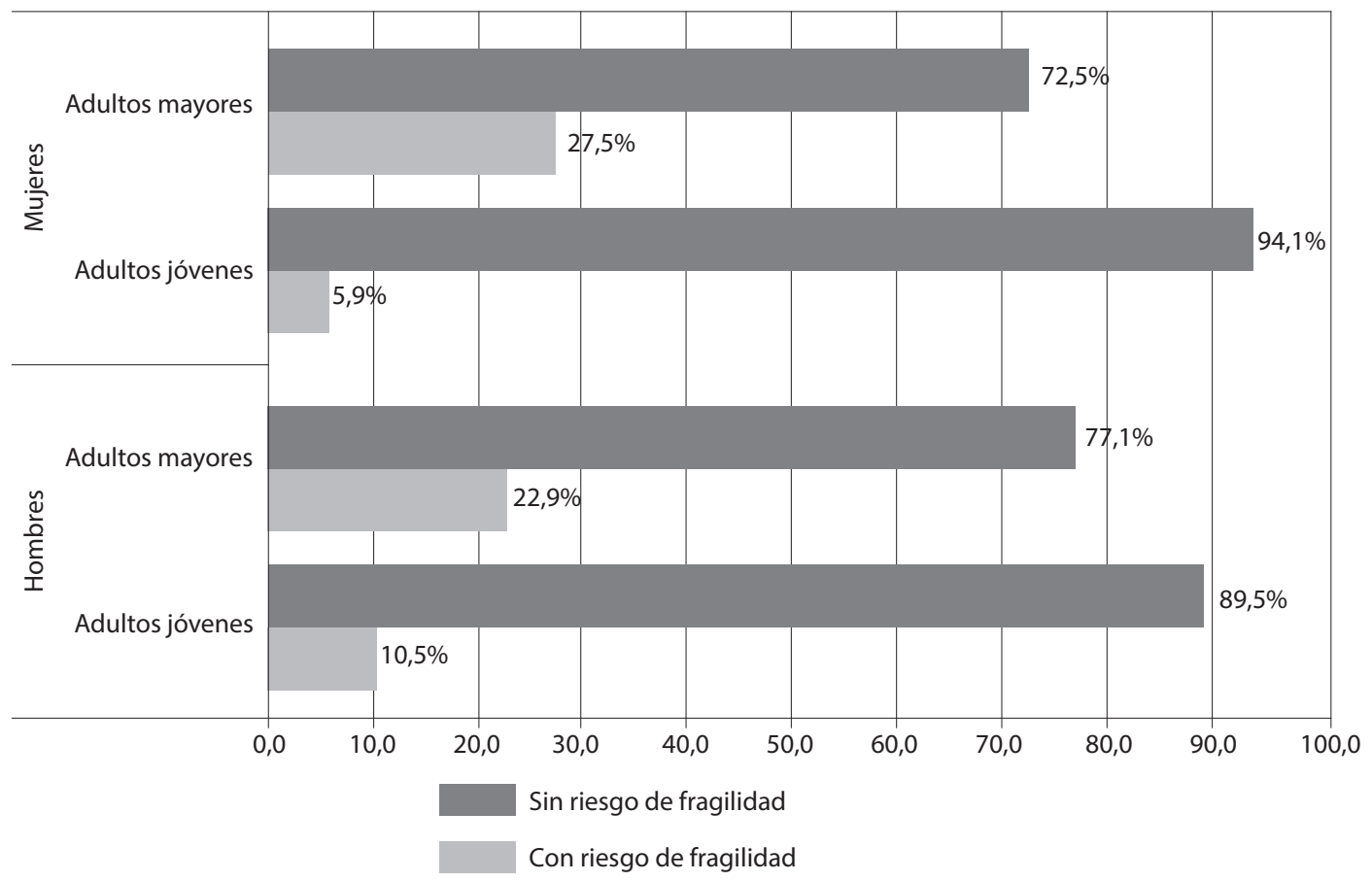

Figura 2. Riesgo de fragilidad en sujetos sometidos a hemodiálisis según edad y sexo. 


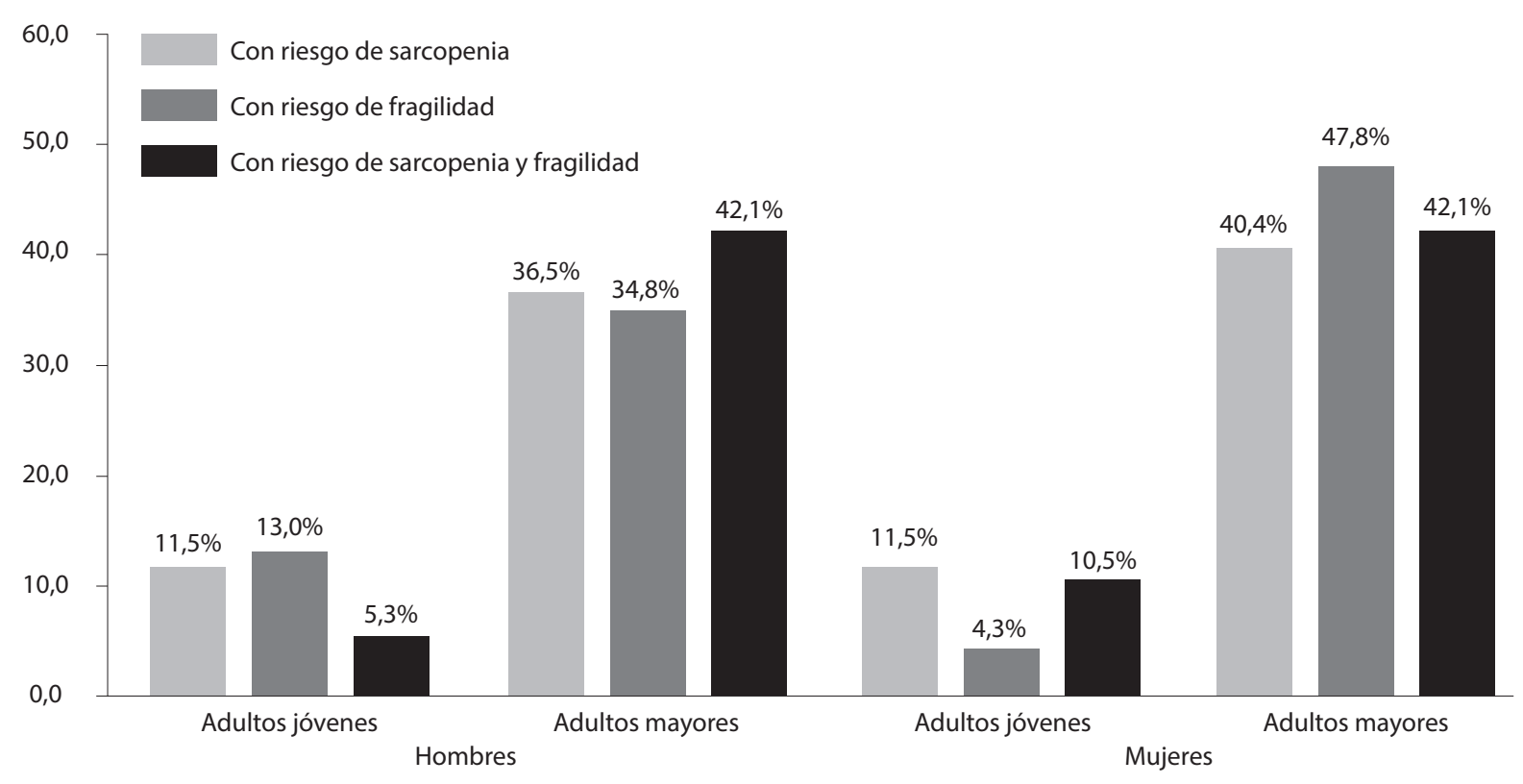

Figura 3. Riesgo de sarcopenia y fragilidad en sujetos sometidos a hemodiálisis según edad y sexo.

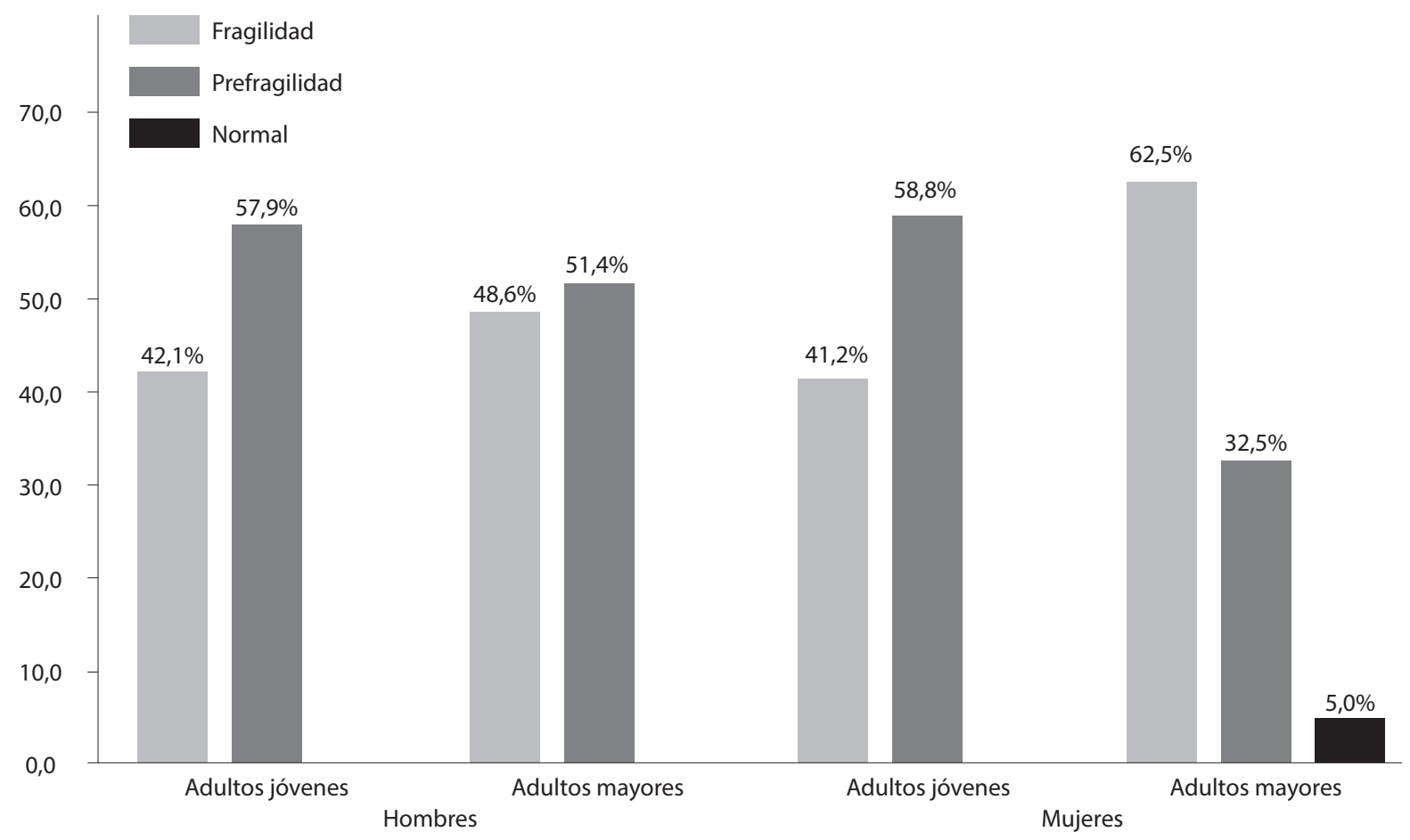

Figura 4. Diagnóstico de fragilidad en sujetos sometidos a hemodiálisis según edad y sexo.

\section{DISCUSIÓN}

La sarcopenia y fragilidad, condiciones relacionadas con el estado nutricional, son frecuentes en sujetos donde la enfermedad es un factor detonante ${ }^{(19)}$. Se conoce que la fragilidad asociada a la sarcopenia aumenta la vulnerabilidad del individuo, la dependencia, el detrimento de la calidad de vida e incremento de la mortalidad 
en la población con enfermedad renal crónica terminal en terapia de hemodiálisis ${ }^{(20,21)}$. Nuestro estudio es el primero en evaluar la sarcopenia y la fragilidad en población peruana con enfermedad renal y hemodiálisis. El principal resultado de este estudio muestra que la asociación de estas dos condiciones es frecuente en pacientes con hemodiálisis (17,1\%). Además, se encontró que dos de cada 10 sujetos presentaron riesgo de fragilidad, siendo más frecuente en mujeres. Los adultos mayores de sexo masculino presentaron sarcopenia con mayor frecuencia $(54,3 \%)$.

Las guías internacionales recomiendan diagnosticar sarcopenia utilizando imágenes y pruebas objetivas de funcionalidad ${ }^{(22)}$. En este estudio se usó la herramienta SARC-F debido a que es un cuestionario previamente validado y de fácil aplicación que facilita la predicción de sarcopenia ${ }^{(23)}$.

En nuestro estudio a todos los pacientes se les realizó tamizaje y diagnóstico de fragilidad. Para el tamizaje se utilizó la escala CFS la cual permite medir la fragilidad. Estudios han demostrado que esta escala hace posible predecir resultados adversos como mortalidad ${ }^{(24)}$ y estancia hospitalaria en adultos mayores ${ }^{(25)}$. Además, se ha demostrado que tiene una alta confiabilidad entre evaluadores (coeficiente de correlación intraclase de $0,97)$ y se correlaciona bien con las medidas objetivas de fragilidad (coeficiente de correlación de Pearson con índice de fragilidad basado en las estadísticas de 0,80$)^{(17)}$. El tamizaje subjetivo debe ser complementado con la evaluación objetiva de la fragilidad. La definición de fragilidad ha sido objeto de controversia y distintas definiciones han surgido. La mayoría de estas incluyen medidas de rendimiento físico o funcionamiento aportadas por el sujeto, algunos indicadores de fatiga o agotamiento y medidas de comorbilidad y discapacidad. La definición más utilizada fue validada en el Cardiovascular Health Study por Fried et al. y se basa en la presencia de al menos tres de cinco criterios ${ }^{(18)}$ y constituye un marco de referencia común para muchos estudios geriátricos.

La sinergia entre la nutrición y la fisioterapia durante la sesión de diálisis podría ser importante, puesto que estudios han informado resultados prometedores relacionados con la mejora de la calidad de vida y el estado nutricional ${ }^{(38,39)}$.

En este estudio se encontró riesgo de fragilidad en $19,8 \%$ de los pacientes, resultado similar a los encontrados por Alfaadhel et al. quienes, en un estudio realizado en pacientes adultos en diálisis, utilizando la escala de CFS, encontraron $25,9 \%$ de riesgo de fragilidad ${ }^{(26)}$.
Clark et al. encontraron una prevalencia estimada de fragilidad en $32,9 \%$ de los pacientes con enfermedad renal terminal, utilizando la misma escala ${ }^{(27)}$. La diferencia con nuestro estudio se puede explicar por el estado avanzado de la enfermedad. En el estudio de Isayere et al. en pacientes adultos mayores, la fragilidad fue de $42,6 \%{ }^{(28)}$, resultados similares a los encontrados en nuestra población adulto mayor utilizando el fenotipo de Fried (44,2 \%) pero mayor según CSF (86,4 $\%)$. Estas diferencias se pueden explicar por los criterios utilizados y el tipo de paciente estudiado.

Nuestro estudio mostró una prevalencia de prefragilidad (según Fried) cercana a 50 \%, ubicándose dentro del rango de resultados encontrados en estudios similares. En estudios en poblaciones norteamericanas ${ }^{(29,30)}$, nipona ${ }^{(31)}$ y egipcia ${ }^{(32)}$ la prefragilidad se presenta entre $32,2 \%$ y $60 \%$.

En este estudio, la fragilidad se diagnosticó en 1 de cada 2 sujetos. Esta cifra supera el rango de $29,8 \%$ a $39,8 \%$ de los estudios realizados en Estados Unidos ${ }^{(30,33)}$, Japón ${ }^{(31)}$ y España ${ }^{(34)}$. Autores como Yadla et al. ${ }^{(35)}$, Johansen et al. ${ }^{(30)}$ y Yoneki et al. ${ }^{(31)}$, mostraron que la prevalencia de fragilidad es mayor en varones que en mujeres $(67,1 \%$ vs $32,9 \% ; 17,1 \%$ vs $14,4 \%$ y $15,9 \%$ vs $14 \%$; respectivamente) contrario a nuestros resultados que encuentran una diferencia de $6,3 \%$ a favor de la población femenina. Además, se ha encontrado en pacientes que reciben terapia sustitutiva renal (en la modalidad de hemodiálisis), una brecha establecida entre adultos mayores y adultos jóvenes menor de $12 \%$ presentado en la población norteamericana ${ }^{(36,37)}$; mientras que en el presente estudio es menor de $25 \%$.

El estudio presenta limitantes. Primero, no se realizó evaluación del estado nutricional. La fragilidad y la sarcopenia son dos condiciones que se asocian al estado nutricional. Se recomienda realizar estudios donde se evalúen estas dos condiciones teniendo en cuenta, además, el diagnóstico de desnutrición asociada a la enfermedad según los nuevos criterios diagnósticos (Global Leadership Initiative on Malnutrition) ${ }^{(16)}$. Otra limitante, fue que no se estudió la severidad de la enfermedad. La fragilidad y la sarcopenia se asocian a la severidad de la enfermedad. Por último, tampoco se evaluó el nivel de actividad física. La sinergia entre la nutrición y la fisioterapia durante la sesión de diálisis puesto que han informado resultados prometedores relacionados con la mejora de la calidad de vida y estado nutricional ${ }^{(38,39)}$. Se deben realizar estudios donde se tengan en cuenta estas variables. 


\section{CONCLUSIONES}

La enfermedad renal crónica terminal se caracteriza por un proceso inflamatorio crónico subclínico que aunado a la toxemia urémica condiciona la aparición de sarcopenia y fragilidad. La presente investigación estableció que 1 de cada 2 sujetos está en riesgo de sarcopenia y tiene el diagnóstico de fragilidad. También, es de notar que ambas condiciones, sarcopenia y fragilidad, se presentaron en un porcentaje importante de pacientes.

Debido a la prevalencia de estas dos condiciones, es necesario nuevos ensayos clínicos randomizados de intervención con fines terapéuticos.

\section{Agradecimientos}

Los autores agradecen a los pacientes del centro de diálisis quienes fueron, son y serán motivo constante de trabajo de calidad; al personal administrativo y asistencial del centro de diálisis quienes permitieron el desarrollo de esta investigación.

\section{Financiación}

El presente estudio no tuvo financiación.

\section{Conflicto de intereses}

Los autores declaran no tener conflicto de intereses.

\section{REFERENCIAS BIBLIOGRÁFICAS}

1. Carrero JJ, Stenvinkel P, Cuppari L, Ikizler A, Kalantar-Zadeh K, Kaysen G, et al. Etiology of the Protein-Energy Wasting Syndrome in chronic kidney disease: A consensus statement from the International Society of Renal Nutrition and Metabolism. J Ren Nutr. 2013;23(2):77- 90.

2. Cobo G, Lindholm B, Stenvinkel P. Chronic inflammation in end-stage renal disease and dialysis. Nephrol Dial Transplant. 2018;33:iii35- iii40.

3. Wang DT, Yang YJ, Huang RH, Zhang ZH, Lin X. Myostatin activates the ubiquitin-proteasome and autophagy-lysosome system contributing to muscle wasting in chronic kidney disease. Oxid Med Cell Longev. 2015; 684965.

4. Sureshbabu A, Ryter SW, Choi ME. Oxidative stress and autophagy: crucial modulators of kidney disease. Redox Biol. 2015;4:208-14.

5. Dikic I. Proteosomal and autophagic degradation systems. Annu Rev Biochem. 2017;86:193- 224.

6. Wang XH, Mitch WE. Mechanism of muscle wasting in chronic kidney disease. Nat Rev Nephrol. 2014;10(9):504-16.

7. Giglio J, Kamimura MA, Lamarca F, Rodrigues J, Santin F, Avesani CM. Association of Sarcopenia with Nutritional
Parameters, Quality of Life, Hospitalization and Mortality Rates of Elderly Patients on Hemodialysis. J Ren Nutr. 2018;28(3):197-207.

8. Kojima G. Prevalence of frailty in end-stage renal disease: a systematic review and meta-analysis. Int Urol Nephrol. 2017;49(11):1989-97.

9. Fielding RA, Vellas B, Evans WJ, Bhansin S, Morley JE, Newman AB, et al. Sarcopenia: an undiagnosed condition in older adults. Current consensus definition: prevalence, etiology and consequences. International working group on sarcopenia. J Am Med Dir Assoc. 2011;12(4):249-56.

10. Chen LK, Liu K, Woo J, Assantachai P, Auyeung TW, Bahyah KS, et al. Sarcopenia in Asian: consensus report of the Asian Working Group for Sarcopenia. J Am Med Dir Assoc. 2014;15(2):95-101.

11. Cruz-Jentoft AJ, Baeyens JP, Bauer JM, Boirie Y, Cederholm T, Landi F, et al. Sarcopenia: European consensus on definition and diagnosis. Age Ageing. 2010;39(4):412-23.

12. Bohm C, Storsley L, Tangri N. The assessment of frailty in older people with chronic kidney disease. Curr Opin Nephrol Hypertens. 2015;24(6):498-04.

13. Ren H, Gong D, Jia F, Xu B, Liu Z. Sarcopenia in patients undergoing hemodialysis: incidence rate, risk factors and its effect on survival risk. Ren Fail. 2016;38(3):364-71.

14. Johansen KL. The frail dialysis population: a growing burden for the dialysis community. Blood Purif. 2015;40(4):288-92.

15. Malmstrom TK, Morley JE. SARC-F: a simple questionnaire to rapidly diagnose sarcopenia. J Am Med Dir Assoc. 2013;14(8):531-2.

16. Rockwood K, Song X, MacKnight C, Bergman H, Hogan $\mathrm{DB}, \mathrm{McD}$ owell I, et al. A global clinical measure of fitness and frailty in elderly people. CMAJ. 2005;173(5):489-95.

17. Fried LP, Tangen CM, Walston J, Newman AB, Hirsch C, Gottdiener J, et al. Frailty in older adults: evidence for a phenotype. J Gerontol A Biol Sci Med Sci. 2001;56(3):148-56.

18. Schlüssel MM, dos Anjos LA, de Vasconcellos MT, Kac G. References values of handgrip dinamometry of healthy adults: a population-based study. Clin Nutr. 2008;27(4):601-7.

19. Cederholm T, Barazzoni R, Austin P, Ballmer P, Biolo G, Bischoff SC, et al. Espen guidelines on definitions and terminology of clinical nutrition. Clin Nutr. 2017; 36(1):49- 64.

20. Hirai K, Ookawara S, Morishita Y. Sarcopenia and Physical Inactivity in Patients with chronic kidney disease. Nephrourol Mon. 2016;8(3):e37443.

21. Isoyama N, Qureshi AR, Avesani CM, Lindholm B, Barany P, Heimbürger $\mathrm{O}$, et al. Comparative associations of muscle mass and muscle strength with mortality in dyalisis patients. Clin J Am Soc Nephrol. 2014;9(10):1720-8.

22. Cederholm T, Jensen GL, Correia MITD, Gonzalez MC, Fukushima R, Higashiguchi T, et al. GLIM criteria for the diagnosis of malnutrition - A consensus report from the global clinical nutrition community. Clin Nutr. 2018; pii: S02615614(18)31344-X. 
23. Malmstrom TK, Miller DK, Simonsick EM, Ferrucci L, Morley JE. SARC-F : a symptom score to predict persons with sarcopenia at risk of poor functional outcomes. J Cachexia Sarcopenia Muscle. 2016;7(1):28 - 36.

24. Wallis SJ, Wall J, Biram RW, Romero-Ortuno R. Association of the clinical frailty scale with hospital outcomes. QJM. 2015;108(12):943-9.

25. Hartley P, Adamson J, Cunningham C, Embleton G, RomeroOrtuno R. Clinical frailty and functional trajectories in hospitalized older adults : A retrospective observational study. Geriatr Gerontol Int. 2017;17(7):1063 - 8.

26. Alfaadhel TA, Soroka SD, Kiberd BA, Landry D, Moorhouse $\mathrm{P}$, Tennankore KK. Frailty and mortality in dialysis: evaluation of a clinical frailty scale. Clin J Am Soc Nephrol. 2015;10(5):832-40.

27. Clark DA, Khan U, Kiberd BA, Turner CC, Dixon A, Landry D, et al. Frailty in end-stage renal disease: comparing patient, caregiver, and clinician perspectives. BMC Nephrol. 2017;18:148.

28. Iyasere OU, Brown EA, Johansson L, Huson L, Smee J, Maxwell AP, et al. Quality of Life and Physical Function in Older Patients on Dialysis: A Comparison of Assisted Peritoneal Dialysis with Hemodialysis. Clin J Am Soc Nephrol. 2016;11(3):423-30.

29. McAdams-DeMarco MA, Law A, Salter ML, Boyarsky B, Gimenez L, Jaar BG, et al. Frailty as a novel predictor of mortality and hospitalization in hemodialysis patients of all ages. J Am Geriatr Soc. 2013;61(6):896-01.

30. Johansen KL, Dalrymple LS, Delgado C, Chertow GM, Segal $\mathrm{MR}$, Chiang J, et al. Factors associated with frailty and its trajectory among patients on hemodialysis. Clin J Am Soc Nephrol. 2017;12(7):1100-8.

31. Yoneki K, Kitagawa J, Hoshi K, Harada M, Watanabe T, Shimoda $\mathrm{T}$, et al. Association between frailty and bone loss in patients undergoing maintenance hemodialysis. J Bone Miner Metab. 2018. [en prensa]

32. Deraz H, Mahmoud AA, Fouad M. Prevalence and correlates of frailty among patients on maintenance hemodialysis. Gen Med (Los Angel). 2015;3:156.

33. Johansen KL, Dalrymple LS, Delgado C, Kaysen GA, Kornark J, Grimes B, et al. Association between body composition and frailty among prevalent hemodialysis patients: A US Renal Data System special study. J Am Soc Nephrol. 2014;25(2):381-9.

34. Bancu I, Graterol F, Bonal J, Fernández-Crespo P, García J, Aguerrevere S, et al. Frail patient in hemodialysis: A new challenge in nephrology - Incidence in our area, Barcelonès Nord and Maresme. J Aging Res. 2017:7624139.

35. Yadla M, John JP, Mummadi M. A study of clinical assessment of frailty in patients on maintenance hemodialysis supported by cashless government scheme. Saudi J Kidney Dis Transpl. 2017; 28(1):15-22.

36. Kutner NG, Zhang R, Huang Y, McClellan WM, Soltow QA, Lea J. Risk factors for frailty in a large prevalent cohort of hemodialysis patients. Am J Med Sci. 2014;348(4):277-82.

37. Johansen KL, Chertow GM, Jin C, Kutner NG. Significance of frailty among dialysis patiens. J Am Soc Nephrol. 2007;18(11):2960-7.

38. Hristea D, Deschamps T, Paris A, Lefrançois G, Collet V, Savoiu C, et al. Combining intra-dialytic exercise and nutritional supplementation in malnourished older haemodialysis patients: Towards better quality of life and autonomy. Nephrology (Carlton). 2016;21(9):785-90.

39. Martin-Alemañy G, Valdez-Ortiz R, Olvera-Soto G, GomezGuerrero I, Aguire-Esquivel G, Cantu-Quintanilla G, et al. The effects of resistance exercise and oral nutritional supplementation during hemodialysis on indicators of nutritional status and quality of life. 2016;31(10):1712-20. 